\title{
Assessing What Information Quality Means in OTS Selection Processes
}

\author{
Claudia Ayala, Xavier Franch \\ Technical University of Catalunya (UPC) \\ \{cayala,franch@lsi.upc.edu\}
}

\begin{abstract}
OTS selection plays a crucial role in the deployment of software systems. One of its main current problems is how to deal with the vast amount of unstructured, incomplete, evolvable and widespread information that highly increases the risks of taking a wrong decision. The goal of our research is to tackle these information quality problems for facilitating the collection, storage, retrieval, analysis and reuse of OTS related information. An essential issue in this endeavor is to assess what OTS selectors mean by Information Quality and their needs to perform an informed selection. Therefore, we are putting forward an on-line survey to get empirical data supporting our approach.
\end{abstract}

\section{Introduction}

Nowadays, the amount of information available about Off-The-Shelf components (hereafter OTS) is vast and still growing. To select OTS (i.e., the process of deciding the appropriate OTS to be integrated in a Component-Based System (CBS)), decision makers have to face not only the current diversity of OTS available in the marketplace, but also the great deal of widespread, heterogeneous, and unstructured information describing each of them [1]. Information Quality (IQ) largely determines the quality of the decisions made, and ultimately affects the quality of the whole software system and its development [2]. Since OTS selectors must rely on the information for their decision-making processes, ensuring IQ is a critical success factor in the development of CBS.

Overtime, librarians and other information professionals have developed a set of criteria to be used to evaluate IQ based on careful experts examination (e.g., authority, format, scope) [3]. However, these criteria are too general and do not provide much guidance to the particular problem of OTS selection. Therefore, IQ is still a critical open issue from the OTS selection perspective [1], [2], [4].

\section{Surveying IQ in OTS Selection Processes}

The goal of our research is to tackle these information quality problems by stating a reference model embracing quality indicators. These quality indicators intend to facilitate the collection, storage, retrieval, analysis and reuse of OTS related information in a quality assurance environment. Such work is based on relevant approaches from the IQ research, several industrial experiences and case studies analyzing OTS selection processes, and interviews run in software companies. Details are found in [5]. However, to enhance our approach, more empirical data is required to assess what IQ means for OTS selectors and their informational needs. With this aim, we are currently performing an empirical study divided in two stages: The first one is addressed by an on-line questionnaire ${ }^{1}$ in order to get as much feedback as possible about OTS IQ experiences. The questionnaire is based on the IQ framework proposed in [6]. The second stage will use the data obtained by the questionnaire to design an interview guide to further explore feasible IQ measures supporting OTS selection.

Participation in the study will be really appreciated. Further details and results of the study may be consulted at http://www.lsi.upc.edu/ cayala/

This work is supported by the Spanish MEC TIN2004-07461-C02-01 project. C. Ayala's work is partially supported by the Agència de Gestió d'Ajuts Universitaris i de Recerca (European Social Fund).

\section{References}

[1] Bertoa, M. et al. "A Survey on the Quality Information Provided by Software Component Vendors" QAOOSE 2003.

[2] Cechich, A., et al. "Trends on COTS Components Identification and Retrieval" ICCBSS 2006.

[3] Boop, R.; Smith, L., Reference and Information Services: An Introduction. Libraries Unlimited, 2000.

[4] Simmons, G.L., Dillon, T.S. "Towards an Ontology for Open Source Software Development" Open Source Systems, Springer, 2006.

[5] Ayala, C., Franch, X., "A Systematic Approach to Manage Information Quality for Supporting Software Package Selection" Research Report. UPC - LSI-07-28-R

[6] Wang, R.Y.; Strong, D.M., "Beyond Accuracy: What Data Quality Means to Data Consumers" Journal of Management of Information Systems, 1996.

\footnotetext{
${ }^{1}$ Freely available at http://www.lsi.upc.edu/ cayala/IQQuestionnaire
} 